\title{
Plantas medicinais utilizadas na medicina popular por moradores da área urbana de Bandeirantes, PR, Brasil
}

\section{Herbal remedies used by residents of the urban areas fron city of Bandeirantes, Paraná, Brazil}

\author{
Simone Beatriz Fuck ${ }^{1 *}$; João Carlos Athanázio ${ }^{2}$; Cristina B. de Lima ${ }^{3}$; Lin Chau Ming ${ }^{4}$
}

Resumo

\begin{abstract}
O presente estudo teve por objetivo levantar o conhecimento a respeito de plantas medicinais dos moradores da área urbana central do município de Bandeirantes-PR. Os dados foram coletados no período de fevereiro a dezembro de 2003, através de entrevistas feitas com mulheres, com idade acima de 40 anos, selecionadas pelo seu conhecimento sobre o uso medicinal de espécies vegetais. As entrevistas foram realizadas através de formulários pré-estruturados, com questões referentes ao entrevistado e à planta citada. Cada entrevista foi registrada com o auxílio de gravador e fotografias para evitar perdas de informações. Foram coletadas amostras vegetais para montagem de exsicatas para posterior identificação botânica. Após a identificação, as exsicatas foram incorporadas ao herbário do Jardim Botânico da Faculdades Luiz Meneghel - FALM, Bandeirantes, PR. Foram registradas 31 espécies vegetais distribuídas em 19 famílias, sendo a Lamiaceae com maior número de espécies, seguida por Rosaceae, Asteraceae e Euphorbiaceae. A parte vegetal mais utilizada na preparação dos remédios foi a folha, e a forma de preparo mais comum foi a infusão. As indicações terapêuticas mais citadas nas entrevistas foram dor de cabeça, diarréia, febre, cólica e infecção.
\end{abstract}

Palavras-chave: Plantas medicinais, medicina popular, área urbana

\begin{abstract}
The present study had the objective of collecting data about herbal remedies from residents of the central urban area fron the city of Bandeirantes, Paraná. The data were collected from February to December 2003, through interviews done with people aging over 40 years old, chosen considering the knowledge about the medical use of herbal species, based on indication done by local residents. The interviews were kept using a form with questions related to the interviewed one and to the herbal medicine mentioned. Each interview was recorded using a cassette recorder and pictures for a later transcription. Vegetal samples were collected as often as possible and the samples were herborized, then exsicats were prepared for later botanical identification. After the identifications, the exsicats were incorporated to the herbaceous border of FALM Botanical Garden (Faculdades Luiz Meneghel, Bandeirantes, Paraná). 31 plants (herbal remedies) were indicated, distributed among 19 families. The Lamiaceae contributed with the highest number of species, followed by Rosacea, Asteraceae and Euphorbiaceae. Leafs were the most used part for the medical preparation and the most common way of preparation was the infusion. The most mentioned therapeutic indications were: headaches, stomachahes, fever, stomach cramps and infection.
\end{abstract}

Key words: Herbal remedies, popular medicine, urban area

1 Mestranda do Curso de Agronomia da Universidade Estadual de Londrina, bolsista do CNPq, email:sbeatriz.fuck@bol.com.br.

2 Prof. Dr. do Curso de Agronomia da Universidade Estadual de Londrina, Centro de Ciências Agrárias, Cep 86051-990, LondrinaPR, email: jcatha@uel.br.

3 FALM - Fundação Faculdades “Luiz Meneghel” - Departamento de Produção Vegetal, Bandeirantes-PR, email: crislima@ffalm.br.

4 FCA/UNESP-Departamento de Produção Vegetal-SP, email: linming@fca.unesp.br.

* Autor para correspondência.

Recebido para publicação 02/12/04 Aprovado em 06/05/05 


\section{Introdução}

As plantas medicinais correspondem, incontestavelmente, as mais antigas armas empregadas no tratamento de enfermidades humanas. A dor fez com que o homem buscasse o analgésico; a doença o remédio, portanto, é fácil inferir que o uso de plantas no combate a doenças seja tão antigo quanto à própria humanidade (OLIVEIRA; AKISUE, 2000).

Houve épocas, entretanto, em que a confiança nas virtudes das ervas diminuiu, contudo, elas mantiveram-se como fonte indispensável de drogas. A indústria química farmacêutica produzia os mais diversos tipos de fármacos, que se mostravam eficazes como medicamentos, porém com custos cada vez mais altos deixando grande parte da população mundial, sem acesso a esses benefícios. Além disso, efeitos colaterais decorrentes do uso desses medicamentos eram cada vez mais freqüentes. Não existia vantagem em se tratar rápida e eficientemente um mal introduzindo-se outro. Os medicamentos precisavam ser ao mesmo tempo eficazes, seguros e de custo acessível a todos (OLIVEIRA; AKISUE, 2000).

Por essas razões, pesquisas sobre espécies vegetais com potencial medicinal vêm sendo revalorizadas, com possibilidade de descoberta de novos princípios ativos úteis como medicamento pela população, e nesse contexto, o chamado "saber popular", é um dos assuntos mais intrigantes dessa pesquisa (DI STASI, 1996).

No Brasil, considerando a ampla diversidade de espécies vegetais, bem como a riqueza étnicocultural, as plantas medicinais devem ocupar posição de destaque em relação à importância do uso popular medicinal. A realização de estudos etnobotânicos possibilita o resgate e a preservação dos conhecimentos populares das comunidades envolvidas (GARLET; IRGANG, 2001).

A compreensão do contexto social e a inclusão de diferentes formas de utilização dos recursos vegetais normalmente têm sido preocupação quase exclusiva dos trabalhadores de etnobotânica que tratam de grupos indígenas específicos. São exemplos típicos os estudos com tribos amazônicas de Balée e Gély (1989), Balick (1988), Anderson e Posey (1985) e Boom (1989) entre outros. As hipóteses, ainda que na maior parte com base em dados qualitativos, esboçam-se na tentativa de compreender a inserção cultural e os mecanismos de utilização das espécies vegetais nas comunidades estudadas. Albuquerque e Chiapetta (1996) e Albuquerque e Andrade (1998) utilizaram essa abordagem no estudo da etnobotânica de grupos afrobrasileiros.

A vasta gama de informações sobre o uso de centenas de plantas como "remédios" em todos os lugares do mundo, leva a necessidade de se desenvolver métodos que facilitem a enorme tarefa de avaliar cientificamente o valor terapêutico de espécies vegetais. Como a maior parte da flora é ainda desconhecida do ponto de vista químico, bem como o saber tradicional associado a esta, predominantemente em países em desenvolvimento, a perda da biodiversidade e o acelerado processo de mudanças cultural acrescentam um censo de urgência em garantir o registro desse saber, inclusive para uso científico (ELISABETSKY, 2000).

A partir desse estudo espera-se resgatar o conhecimento popular sobre plantas medicinais, registrando informações básicas para posterior estudos fármacos, pois, muitas espécies utilizadas para fins terapêuticos pela população tiveram, comprovação de sua ação medicinal em estudo de laboratório (MARQUESINI, 1995).

Muitas plantas são conhecidas popularmente pelo nome vulgar, o que dificulta a sua identificação correta, podendo-se citar como exemplo o boldo. A espécie Peumus boldus Molina, originária do Chile, contém alcalóides especificamente boldina e óleo essencial, destacando-se o ascariol, apresentando a ação colerética e colagoga. No Brasil, diversas espécies sem ação farmacológica comprovada são conhecidas e comercializadas como boldo, tendo-se 
como exemplo Coleus barbatus (Andr.) Benth, do qual foram realizados alguns estudos químicos e farmacológicos, mas as ações colerética e colagoga não foram comprovadas (BACCHI, 1996). Sendo assim, a identificação botânica é essencial nesse tipo de trabalho, evitando-se o uso de plantas inócuas até uma intoxicação por espécies venenosas (BOTSARIS, 1995).

Além do resgate cultural, esse tipo de levantamento tem como benefício o retorno a população de resultados seguros, valorizando e recolocando, com maior ênfase as informações experimentadas e aprovadas geração após geração (LIMA, 2000).

O presente estudo, tem por objetivo levantar o conhecimento a respeito de plantas medicinais dos moradores da área urbana central do Município de Bandeirantes-PR, bem como registrar dados relacionados ao preparo, indicações de uso, coleta e identificação das espécies citadas.

\section{Material e Métodos}

Os dados foram coletados no período de Fevereiro a Dezembro de 2003 na área urbana central do Município de Bandeirantes-PR, através de entrevistas feitas a cinco informantes mulheres, com idade média acima de 40 anos, selecionadas devido a seu conhecimento sobre o uso medicinal de espécies vegetais, entre os moradores locais por um levantamento etnobotânico prévio.

As entrevistas foram realizadas utilizando-se formulários pré-estruturados (MARTIN, 1995) com questões referentes ao entrevistado (nome, sexo, idade, etc) e a planta citada (nome popular, uso, parte empregada etc,). Cada entrevista foi registrada com o auxílio de um gravador e fotografias para posterior transcrição, evitando-se perda de informações durante as mesmas.

Sempre que possível e permitido pelo informante, coletou-se amostra vegetal. Cada material foi herborizado, com auxílio de prensa e jornal, levado a estufa com temperatura e tempo de secagem apropriados, seguindo-se a montagem da exsicata para posterior identificação botânica de acordo com as indicações de Ming (1996). Após a identificação, as exsicatas foram incorporadas ao herbário do Jardim Botânico da FALM - Faculdades Luiz Meneghel - Bandeirantes-PR.

\section{Resultados e Discussão}

Os informantes selecionados foram todas mulheres, fato este analisado de várias formas, por exemplo, Dias (1999) relacionou as diferenciações do conhecimento e uso entre os sexos, com as atividades diárias desempenhadas pelos moradores, pois, em seu estudo, na maioria das famílias entrevistadas, a mulher era a responsável pelo cultivo e preparo das plantas medicinais, assim como pela alimentação e cuidados dispensados as crianças e outros familiares quando enfermos. Segundo Amorozo e Gély (1988), existe uma certa diferenciação entre o conhecimento do homem e da mulher com relação às plantas que crescem em ambientes manejados ou não. De modo geral, a mulher domina melhor o conhecimento das plantas que crescem próximo a sua residência, no quintal e no sitio, enquanto o homem conhece mais as plantas do mato. Mas esta especialização não é de modo algum rígida, algumas mulheres conhecem os "remédios do mato" tão bem como seus maridos. Ming (1995) interpreta que esse número pode estar relacionado ao local da entrevista e a atividade dos entrevistados.

Quanto à faixa etária, verifica-se que todas as entrevistadas têm acima de 40 anos. Lima (2000) relata que, pessoas a partir de 30 anos foram as que mais se dispuseram a conversar e fornecer informações sobre plantas medicinais, os mais jovens disseram preferir remédios quimiossintetizados e demonstraram-se incrédulos no "poder de cura das plantas medicinais". 
Foram indicadas 31 plantas, distribuídas em 19 famílias (Tabela 1), sendo que Lamiaceae contribuiu com maior número de espécies $(26,32 \%)$, seguida por Rosaceae, Asteraceae e Euphorbiaceae com $15,79 \%$ cada uma. Marodin (2002) ressaltou que as famílias Lamiaceae e Asteraceae ocupam as primeiras posições nos levantamentos feitos na região Sul do Brasil.

A parte vegetal mais citada como utilizada na preparação dos remédios foi a folha, seguida por raiz, pétalas e planta inteira. (Tabela 1) resultado análogo pode ser observado em diversos trabalhos como o de Kubo (1997), Dias (1999), Grams (1999), Souza (2000) e Lima (2000).

A infusão (popularmente denominada de chá) destaca-se como modo de preparo mais recomendado (Tabela 1) e, de acordo com Castellani (1999), este é utilizado em todas as partes de plantas medicinais tenras tais como folhas, botões e flores, pois as mesmas são ricas em componentes voláteis, aromas delicados e princípios ativos que se degradam pela ação combinada da água e do calor prolongado.

Em relação as indicações terapêuticas, há casos em que essas referem-se a diferentes usos como: sintoma de determinada doença (dor de cabeça, dor de barriga, febre, cólica e infecção) e não a doença propriamente dita; noutros, a própria doença é alvo da indicação (gripe, labirintite e pressão alta). Algumas indicações dizem respeito aos efeitos esperados com a utilização do remédio, como no caso das menções para depurativo, calmante, emagrecedor e vermífugo. Existem ainda, aquelas nas quais os órgãos que se pretende atingir com o tratamento (garganta, rins, intestino, estômago, olhos, ouvido, pele, cabelo, etc) são lembrados. Este elenco de itens revela, por conseqüência, não haver discriminação entre sintomas e doenças pelos entrevistados, concordando com os resultados descritos nas pesquisas de Kubo (1997), Dias (1999), Grams (1999) e Lima (2000).

Apesar da herança cultural ter sido a maior fonte de aprendizagem a respeito de plantas com utilização medicinal, existe um grande interesse em adquirir maiores informações sobre o uso e cultivo dessas plantas por parte das entrevistadas, que invariavelmente recorrem a fontes externas como livros, revistas e programas de televisão, indicando haver uma forte influência desses meios em seus conhecimentos. Assim, instituições de pesquisa podem contribuir para a disseminação dessas informações desenvolvendo e divulgando de forma prática e acessível os resultados obtidos em estudos complementares nessa área.

Tabela 1. Listagem das plantas com nome popular, família, nome cientifico, uso terapêutico, modo de preparo e parte da planta utilizada indicadas pelos moradores da área urbana do município de Bandeirantes - PR.

\begin{tabular}{|c|c|c|c|c|c|}
\hline $\begin{array}{l}\text { Nome } \\
\text { popular }\end{array}$ & Família & Espécie & Uso terapêutico & Modo de preparo & $\begin{array}{c}\text { Parte } \\
\text { utilizada }\end{array}$ \\
\hline Alecrim & Lamiaceae & Rosmarinus officinalis & Cicatrizante e calmante. & $\begin{array}{l}\text { Macera a folha, côa e coloca sobre o } \\
\text { ferimento. Chá com as folhas. }\end{array}$ & $\mathrm{F}$ \\
\hline $\begin{array}{l}\text { Ameixa } \\
\text { Amarela }\end{array}$ & Rosaceae & Eryobotria japonica & $\begin{array}{l}\text { Controla a pressão para não } \\
\text { abaixar muito. }\end{array}$ & $\begin{array}{l}\text { Chá com } 2 \text { folhas secas à sombra, } \\
\text { misturada com } 8 \text { a } 10 \text { folhas de pitanga }\end{array}$ & $\mathrm{F}$ \\
\hline Arnica & Asteraceae & Porophyllum ruderale & $\begin{array}{l}\text { Antiflamatório e para } \\
\text { desinchar as juntas. }\end{array}$ & $\begin{array}{l}\text { Curte no álcool, molha um pano e } \\
\text { coloca sobre o machucado }\end{array}$ & $\mathrm{F}$ \\
\hline Arruda & Rutaceae & Ruta graveolens L. & $\begin{array}{l}\text { Dor de ouvido, gripe e } \\
\text { pressão alta. }\end{array}$ & $\begin{array}{l}\text { Macera e côa } 5 \text { folhas e pinga no } \\
\text { ouvido. Chá com } 5 \text { folhas tomar frio }\end{array}$ & $\mathrm{F}$ \\
\hline Babosa & Liliaceae & $\begin{array}{l}\text { Aloe vera } \mathrm{L} . \\
\text { Aloe arborescens }\end{array}$ & Gastrite. & $\begin{array}{l}\text { Corta a folha em pedaços e deixa em } \\
\text { um copo com água por alguns minutos, } \\
\text { depois beba. }\end{array}$ & $\mathrm{F}$ \\
\hline
\end{tabular}


continuação

\begin{tabular}{|c|c|c|c|c|c|}
\hline $\begin{array}{l}\text { Nome } \\
\text { popular }\end{array}$ & Família & Espécie & Uso terapêutico & Modo de preparo & $\begin{array}{c}\text { Parte } \\
\text { utilizada }\end{array}$ \\
\hline Balsamo & Crassulaceae & Sedum madagascariense & Gastrite, afta e cicatrizante. & $\begin{array}{l}\text { Bater } 5 \text { folhar com leite e beber em } \\
\text { jejum para gastrite, cortar a folha e } \\
\text { colocar sobre o ferimento. }\end{array}$ & $\mathrm{F}$ \\
\hline Boldo & Lamiaceae & Plectranthus barbatus & Estômago e ressaca. & Macerar 5 folhas em água fria & $\mathrm{F}$ \\
\hline Cânfora & Asteraceae & Artemísia canphorata & Pâncreas, nervosismo e tpm. & Chá com as pontas das folhas & $\mathrm{F}$ \\
\hline $\begin{array}{l}\text { Capim } \\
\text { Cidreira }\end{array}$ & Poaceae & Cymbopogon citratus & Calmante. & Chá com umas 3 folhas. & $\mathrm{F}$ \\
\hline $\begin{array}{l}\text { Erva } \\
\text { Cidreira }\end{array}$ & Verbenaceae & Lippia alba & Calmante, gripe e febre. & Chá com as folhas & $\mathrm{F}$ \\
\hline $\begin{array}{l}\text { Erva de } \\
\text { Santa } \\
\text { Maria }\end{array}$ & Chenopodiaceae & Chenopodium ambrosioides & Vermifugo. & Chá com as folhas & $\mathrm{F}$ \\
\hline $\begin{array}{l}\text { Erva doce } \\
\text { ou Funcho }\end{array}$ & Umbeliferae & Foeniculum vulgare & Calmante. & Chá com as sementes e as folhas & $\mathrm{F}$ e $\mathrm{S}$ \\
\hline $\begin{array}{l}\text { Espinheira } \\
\text { Santa }\end{array}$ & Celastraceae & Maytenus ilicifolia & Rins e gastrite. & Chá com 5 a 6 folhas em 1 litro de água & $\mathrm{F}$ \\
\hline Gengibre & Zingiberaceae & Zingiber officinale & Expectorante. & $\begin{array}{l}\text { Cortar a raiz em finas fatias e curtir no } \\
\text { mel, depois só tomar o mel. }\end{array}$ & $\mathrm{R}$ \\
\hline Gerânio & Geraniaceae & Pelargonium $\mathrm{sp}$ & $\begin{array}{l}\text { Surdez e limpeza dos } \\
\text { pulmões. }\end{array}$ & $\begin{array}{l}\text { Chá com } 3 \text { ou } 4 \text { folhas pro pulmão, } \\
\text { macerar e coar a folha e pingar no } \\
\text { ouvido. }\end{array}$ & $\mathrm{F}$ \\
\hline Guiné & Phytolaccaceae & Petiveria alliacea & Dor nas Juntas. & Curtir no álcool algumas folhas & $\mathrm{F}$ \\
\hline Hortelã & Lamiaceae & Mentha pulegium & $\begin{array}{l}\text { Calmante para criança e } \\
\text { queda de cabelo. }\end{array}$ & $\begin{array}{l}\text { Chá com algumas folhas para criança e } \\
\text { macerar a folha retirando o suco e } \\
\text { passar com algodão no couro cabeludo. }\end{array}$ & $\mathrm{F}$ \\
\hline Mandioca & Euphorbiaceae & Manihot esculenta & Coceira no corpo. & $\begin{array}{l}\text { Chá com } 3 \text { ou } 4 \text { folhas e jogar sobre o } \\
\text { corpo. }\end{array}$ & $\mathrm{F}$ \\
\hline Manga & Anacardiaceae & Mangifera indica & Labirintite & $\begin{array}{l}\text { Colocar de } 8 \text { a } 10 \text { folhas no travesseiro } \\
\text { para dormir. }\end{array}$ & $\mathrm{F}$ \\
\hline Mertiolate & Euphorbiaceae & Jatropha $\mathrm{sp}$ & Cicatrização & $\begin{array}{l}\text { Quebra o galho e coloca o sumo no } \\
\text { machucado }\end{array}$ & $\mathrm{F}$ e $\mathrm{C}$ \\
\hline Pitanga & Myrtaceae & Eugenia uniflora & Pressão alta e reumatismo & $\begin{array}{l}\text { Chá com } 10 \text { folhas de pitanga e } 2 \text { folhas } \\
\text { secas de ameixa amarela. }\end{array}$ & $\mathrm{F}$ \\
\hline Poejo & Lamiaceae & Mentha pulegium & Febre em crianças. & $\begin{array}{l}\text { Chá com } 4 \text { ou } 5 \text { folhas, se a criança for } \\
\text { maior pode colocar mais folhas. }\end{array}$ & $\mathrm{F}$ \\
\hline $\begin{array}{l}\text { Quebra- } \\
\text { Pedra }\end{array}$ & Euphorbiaceae & Phyllanthus corcovadensis & Dor na bexiga e rins. & Chá com a planta inteira. & $\mathrm{Pi}$ \\
\hline Romã & Punicaceae & Punica granatum & $\begin{array}{l}\text { Infecção de garganta e } \\
\text { diarréia }\end{array}$ & $\begin{array}{l}\text { Chá com } 2 \text { a } 3 \text { pontas de folha para } \\
\text { garganta, chá com a casca de } 1 \text { fruta } \\
\text { pequena para cortar diarréia. }\end{array}$ & $\mathrm{F}$ e Cc \\
\hline $\begin{array}{l}\text { Rosa } \\
\text { Branca }\end{array}$ & Rosaceae & Rosa sp & $\begin{array}{l}\text { Limpeza de útero e } \\
\text { corrimento }\end{array}$ & $\begin{array}{l}\text { Chá com as pétalas de uma rosa, } 5 \\
\text { folhas de seni e raiz de salsa }\end{array}$ & $\mathrm{P}$ \\
\hline $\begin{array}{l}\text { Rosa } \\
\text { Vermelha }\end{array}$ & Rosaceae & Rosa sp & Descansa os olhos & $\begin{array}{l}\text { Chá com as pétalas de uma rosa grande } \\
\text { para lavar os olhos }\end{array}$ & $\mathrm{P}$ \\
\hline Salsa & Apiaceae & Petroselinum crispum & $\begin{array}{l}\text { Limpeza de útero e } \\
\text { corrimento }\end{array}$ & $\begin{array}{l}\text { Chá com a raiz misturado a } 5 \text { folhas de } \\
\text { seni e pétalas de rosa branca }\end{array}$ & $\mathrm{R}$ \\
\hline Salvia & Lamiaceae & Salvia officinalis & $\begin{array}{l}\text { Gripe, febre, estomago e dor } \\
\text { de cabeça }\end{array}$ & Chá com as folhas sem a ponta. & $\mathrm{F}$ \\
\hline Seni & Caesalpinaceae & Senna $\mathrm{sp}$ & $\begin{array}{l}\text { Laxante, emagrecer, } \\
\text { infecção ovário, corrimento } \\
\text { e dor na bexiga }\end{array}$ & Chá com 8 a 10 folhas. & $\mathrm{F}$ \\
\hline Tansagem & Plantaginaceae & Plantago lanceolata & Infecção de garganta & Chá com a planta inteira e tomar frio & $\mathrm{Pi}$ \\
\hline $\begin{array}{l}\text { Cravo de } \\
\text { defunto }\end{array}$ & Asteraceae & Tagetes minuta & $\begin{array}{l}\text { Depurativo do sangue, pele e } \\
\text { ferida brava }\end{array}$ & $\begin{array}{l}\text { Chá com } 2 \text { pontas de folhas, também } \\
\text { jogar sobre o corpo }\end{array}$ & $\mathrm{F}$ \\
\hline
\end{tabular}

$\mathrm{F}=$ Folha $; \mathrm{Fl}=$ Flor $\mathrm{P}=$ pétala $; \mathrm{P}=$ Planta inteira $\mathrm{C}=$ Caule $\mathrm{Cc}=\mathrm{Casca} ; \mathrm{R}=$ Raiz . 


\section{Referências}

AMOROZO, M. C. M.; GELY, A. L. Uso de plantas medicinais por caboclos do Baixo amazonas, BarcarenaPA, Brasil. Boletim do Museu Paraense Emílio Goeldi, Série Botânica, Belém, v. 4, p. 47-131, 1988.

ANDERSON, A. B. W.; POSEY, D. A. Manejo de cerrado pelo índios Kayapó. In: Boletim do Museu Paraense Emilio Goeldi, Botanica, 2(1), SCT/CNPq/MPEG, 1985. p. 77-98

ALBUQUERQUE, U. P.; CHIAPETTA, A A. Contribuição etnobôtanica para o universo ritual dos cultos afrobrasileiros. In. LIMA, T. (Org.). Sincretismo religioso: o ritual afro. Recife: Massangana, 1996. p. 188-197.

ALBUQUERQUE, U. P.; ANDRADE, L. H. Etnobotânica del gênero Ocimum L. (Lamiaceae) em lãs comunidades afrobrasilenas. Anales Del Jardín Botânico de Madrid, Madrid, v. 56, p. 106-118, 1998.

BACCHI, E. M. Controle de qualidade de fitoterápicos. In: DISTASI, L. L. (org.) Plantas medicinais: arte e ciência. Um guia de estudo interdisciplinar. São Paulo: Editora da Universidade Estadual Paulista, p. 169-186. 1986.

BALÉE, W.; GÉLY, A. Maneged forest succession in Amazonia: the Ka'apor case. In: Rescurce Management in Amazonia: indigenousand folks strategies. Posey, D. A. and Balée, W. (editors), New York, EUA, The New York Botanical Garden, 1989. p. 129-158.

BALICK, M. I. The use of palms by the Apinayé and Guajajara indians of northeastern Brazil. In: The palmtree of life: biology, utilization and conservation. Balick, M. J. (editor) New York, EUA, Advances in Economy Botany, v. 6 / NYBG, 1988. p. 65-90

BOTSARIS, A. S. Fitoterapia chinesa e plantas brasileiras. São Paulo: Ícone, 1995. p. 550

CASTEllani, D. C. Plantas medicinais. Viçosa: Agromídia software, 1999.

DIAS, M. C. Plantas medicinais utilizadas no distrito de Juquiratiba - Municipio de Conhas - SP. 1999. 82 p. Dissertação (Mestrado em Agronomia) - Universidade Estadual Paulista, Botucatu, 1999.

DI STASI, L. C. (Org). Plantas medicinais: arte e ciência. São Paulo: Unesp, 1996. p. 230.

ELISABETSKY, E. Etnofarmacologia como ferramenta na busca de substâncias ativas. In: SIMÕES, C. M. O. (Org.). Farmacognosia: da planta ao medicamento. 2. ed. Florianópolis: UFSC, 2000. p. 87-99.
GARLET, T.M. B.; IRGANG, B. E. Plantas medicinais utilizadas na medicina popular por mulheres trabalhadoras rurais de Cruz Alta, Rio Grande do Sul, Brasil. Revista Brasileira de Plantas Medicinais, Botucatu, v. 4, p. 9-18, 2001.

GRAMS, W. F. M. P. Plantas medicinais de uso popular em cinco distritos da ilha de Santa Catarina, Florianópolis, SC. 1999. 160 p. Dissertação (Mestrado em Botânica) - Setor de Ciências Biológicas, Universidade Federal do Paraná, Curitiba, 1999.

KUBO, R. R. Levantamento das plantas de uso medicinal, em Coronel Bicaco, RS. 1997. 160 p. Dissertação (Mestrado em Botânica) - Universidade Federal do Rio Grande do Sul, Porto Alegre, 1997.

LIMA, C. B. Plantas medicinais utilizadas em duas localidades do município de Bandeirantes-PR. 2000. 103 p. Dissertação (Mestrado em Agronomia) - Universidade Estadual Paulista, Botucatu, 2000.

MARODIN, S. M. Plantas medicinais no Município de Dom Pedro de Alcântara, Estado do Rio Grande do Sul, Brasil: espécies, famílias e usos em três grupos da população humana. Revista Brasileira de Plantas Medicinais, Botucatu, v. 5, p.1-9, 2002.

MARQUESINI, N. R. Plantas usadas como medicinais pelos índios do Paraná e Santa Catarina, Sul do Brasil. 1995. 290 p. Dissertação (Mestrado em Botânica) Setor de Ciências Biológicas, Universidade Federal do Paraná, Curitiba, 1995.

MARTIN, G. J. Ethnobotany: a methods manual. London: Chapmam \& Hall, 1995. 268 p.

MING, L. C. Coleta de plantas medicinais. In: DI STASI, L.C. (Org.). Plantas medicinais: arte e ciência. São Paulo: UNESP, 1996. p. 47-68.

MING, L. C. Plantas medicinais utilizadas pelos seringueiros na Reserva Extrativista "Chico Mendes", Acre, Brasil. 1995. 180 p. Tese (Doutorado em Botânica) Instituto de Biociências, Universidade Estadual Paulista, Botucatu, 1995.

OLIVEIRA, F.; AKISSUE, G. Fundamentos de Farmacobotânica. 2. ed. São Paulo: Atheneu, 2000. 178 p.

SOUZA, J. M. A. Plantas medicinais utilizadas por seringueiros do projeto assentamento extrativista São Luis do Remanso, Acre. 2000. 111 p. Dissertação. (Mestrado em Agronomia) - Universidade Estadual Paulista, Botucatu, 2000. 